LA-UR-02- 4903

Approved for public release;

distribution is unlimited.

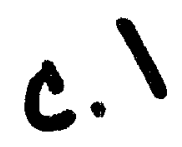

\author{
Title: \\ MAGNETIC DESIGN OF LARGE-BORE \\ SUPERCONDUCTING QUADRUPOLES FOR THE AHF
}

Author(s):

Andrew J. Jason, LANSCE-1

Peter L. Walstrom, LANSCE-1

Nikolai Andreev, Fermi Nat. Accel. Lab.

Vladimir S. Kashikhin, Fermi Nat. Accel. Lab.

Peter J. Limon, Fermi Nat. Accel. Lab.

Vadim V. Kashikhin, Fermi Nat. Accel. Lab.

John Tompkins, Fermi Nat. Accel. Lab.

Submitted to:

International Cryogenic Engineering Conference

Grenoble, France

July 22-26, 2002

Los Alamos National Laboratory, an affirmative action/equal opportunity employer, is operated by the University of California for the U.S. Department of Energy under contract W-7405-ENG-36. By acceptance of this article, the publisher recognizes that the U.S. Government retains a nonexclusive, royalty-free license to publish or reproduce the published form of this contribution, or to allow others to do so, for U.S. Government purposes. Los Alamos National Laboratory requests that the publisher ident $\equiv$ s article as work performed under the auspices of the U.S. Department of Energy. Los Alamos National Laboratory strongly sup $\square$ academic freedom and a researcher's right to publish; as an institution, however, the l.aboratory does not endorse the viewpoint of a publication or guarantee its technical correctness. 


\title{
Magnetic Design of Large-Bore Superconducting Quadrupoles for the AHF
}

\author{
Nikolai Andreev, Vladimir S. Kashikhin, Member, IEEE, Vadim V.Kashikhin, Peter J. Limon, \\ John Tompkins, Fermilab
}

Andrew Jason, Peter Walstrom, LANL

\begin{abstract}
The Advanced Hydrotest Facility (AHF), under study by LANL, utilizes large-bore superconducting quadrupole magnets to image protons for radiography of fast events. In this concept, 50-GeV proton bunches pass through a thick object and are imaged by a lens system that analyzes the scattered beam to determine object details. Twelve simultaneous views of the object are obtained using multiple beam lines. The lens system uses two types of quadrupoles: a large bore $(48 \mathrm{~cm}$ beam aperture) for wide field of view imaging and a smaller bore $(23 \mathrm{~cm}$ aperture) for higher resolution images. The gradients of the magnets are 10.14 T/m and $18.58 \mathrm{~T} / \mathrm{m}$ with magnetic lengths of $4.3 \mathrm{~m}$ and 3.0 $m$, respectively. The magnets are sufficiently novel to present a design challenge. Evaluation and comparisons were made for various types of magnet design: shell and racetrack coils, cold and warm iron, as well as an active superconducting screen. $\mathrm{Nl}_{3} \mathrm{Sn}$ cable was also considered as an alternative to avoid quenching under high beam- scattering conditions. The superconducting shield concept eliminates the iron core and greatly lessens the cryogenic energy needed for cool down. Several options are discussed and comparisons are made.
\end{abstract}

Index Terms - Superconducting Magnets, Quadrupole,Large Bore, AHF, Magnetic Design.

\section{INTRODUCTION}

$\mathrm{T}$ HE Advanced Hydrotest Facility (AHF) [1], under study by LANL, utilizes large-bore superconducting quadrupole magnets to image protons for radiography of fast events [2]. The magnetic optics of this facility is based on large bore quadrupole magnets. The large quantity $(\sim 84)$ and large length $3 \mathrm{~m}-4 \mathrm{~m}$ of this magnet are the reasons careful optimization of all steps for magnet design.

One of the challenges in this design is obtaining a high quality $\left(10^{-4}\right)$ quadrupole (magnetic) field in $100 \%$ aperture. This follows from the beam optics aberration analysis [3].

Another challenge for the magnet design is a severe space limitation in magnet width. Because quadrupoles will be installed very close to each other, no fringing fields will be allowed. The limited space for return flux in the median plane directs the design to a "Figure-8" type quadrupole.

Manuscript received August 4, 2002.

N.Andreev, V.S.Kashikhin, V.V.Kashikhin, P.J.Limon, J.Tompkins with FERMILAB, Batavia, IL 60510, MS 316, USA. E-mail: kaslı@fnal.gov.

A.Jason, P.Walstrom with LANL.
One of the options to reduce magnet weight and eliminate fringing fields is to use an active shielding winding. The lower magnet weight and dimensions compensate the larger quantity of superconductor.

The rather attractive option is to have a cold iron core which help to reduce the quantity of superconductor but iron saturation effects should be carefully investigated.

The large magnetic forces of $\sim 300 \mathrm{t} / \mathrm{m}$ can cause problems with mechanical stability. The racetrack type coils can accommodate these forces easier than shell type coils but have larger dimensions.

Because of rather high magnetic field $6 \mathrm{~T}-8 \mathrm{~T}$ in the area of superconducting coils the proper choice of superconductor $\mathrm{NbTi}$ or $\mathrm{Nb}_{3} \mathrm{Sn}$ also should be investigated.

\section{BASIC DESIGN PARAMETERS}

The project is oriented towards lowering overall magnet system cost, achieving a high field quality, and using existing superconducting magnet manufacturing technologies.

There are two types of superconducting quadrupoles to work with different image resolution.

TABLE I

Main Quadrupole Parameters

\begin{tabular}{|c|c|c|}
\hline Parameter & $\begin{array}{c}\text { Large } \\
\text { Quadrupole }\end{array}$ & $\begin{array}{c}\text { Small } \\
\text { Quadrupole }\end{array}$ \\
\hline Nominal quadrupole gradient, $T / m$ & 10.14 & 18.58 \\
\hline Maximum quadrupole gradient, $\mathrm{T} / \mathrm{m}$ & 13.18 & 24.15 \\
\hline Aperture diameter, $\mathrm{mm}$ & 482.6 & 228.6 \\
\hline Magnetic length, m & 4.3 & 3.0 \\
\hline $\begin{array}{l}\text { Magnetic field nonlinearity, \% } \\
\text { at the reference radius, } \mathrm{mm}\end{array}$ & $\begin{array}{l}\leq 0.01 \\
241.3\end{array}$ & $\begin{array}{l}\leq 0.01 \\
114.3\end{array}$ \\
\hline Total quadrupoles width, $m$ & \multicolumn{2}{|c|}{1.92} \\
\hline LHe Cooling, K & 4.2 & 4.2 \\
\hline
\end{tabular}

The total width of both quadrupoles should not exceed $1.92 \mathrm{~m}$ in most narrow space between magnets. The width of each quadrupole can be estimated taking into account the same magnetic resistance for the return flux and relations between winding radiuses and gradients as : 


$$
\begin{aligned}
& R o u l \cong \frac{\frac{D}{2}-R w s+R w l \times \frac{G s}{G l} \times\left(\frac{R w s}{R w l}\right)^{2}}{1+\frac{G s}{G l} \times\left(\frac{R w s}{R w l}\right)^{2}} \\
& \text { Roul }+ \text { Rous }=\frac{D}{2}=\text { Const }
\end{aligned}
$$

where $D$, total quadrupoles cold mass width, $R_{\text {oul }}, R_{\text {ous }}$ - outer cold mass radiuses of large and smaller quadrupoles $R_{w l}, R_{w s}$ and $G_{l}, G_{s}$ are main winding radiuses and magnetic field gradients.

\section{QUADRUPOLE MAGNETIC DESIGN ANALYSIS}

There are several well known superconducting magnets design concepts, which are used in superconducting magnets for particle accelerators. Superconducting magnets of Tevatron, (Fermilab) are based on a warm iron yoke design, while RHIC, (BNL) are based on cold iron yoke design. The superferric magnet concept takes place when cold iron is close to the magnet aperture and substantially influences on the magnetic field level and quality.

The main differences for the large bore quadrupoles design are large quadrupole dimensions, large total magnetic flux and tight limits at the magnet width. In this case, a magnet with active shielding magnetic field winding also should be investigated.

\section{A. Quadrupoles With Warm Iron Yoke}

The warm iron core quadrupole has a low cold mass, inexpensive iron core and rather low iron saturation effects. The quadrupole cross-section is shown on Fig.1. The windings of large and small-bore quadrupoles were designed using ROXIE[4]. The iron yoke design and field quality analysis was made by OPERA2D [5]. The calculated quadrupole parameters are shown in Tab.II.

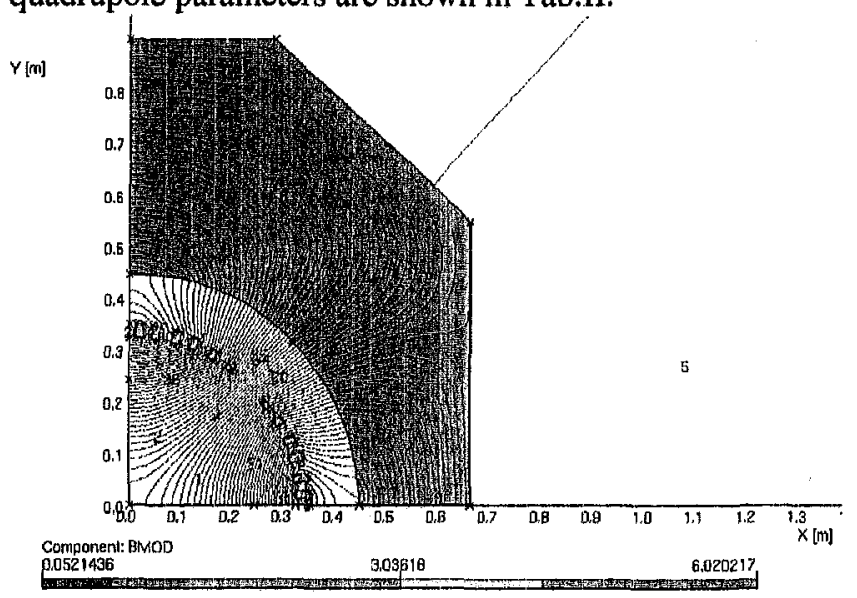

Fig.1. Flux lines distribution in the magnet with warm yoke
TABLE II

QUADRUPOLE WITH WARM YOKE

\begin{tabular}{lcc}
\hline Parameter & Large Quadrupole & Small Quadrupole \\
\hline Total current/pole, kA & 903 & 674 \\
Magnetic flux density, T & 3.25 & 3.22 \\
at reference radius,mm & 241.3 & 114.3 \\
Maximum coil flux density, T & 6.0 & 7.14 \\
Maximum iron flux density, T & 2.8 & 2.92 \\
Energy of magnetic field, MJ/m & 2.14 & 1.08 \\
Magnetic forces/half-coil: & & \\
Fx, t/m & 147 & 108 \\
Fy, t/m & -156 & -140 \\
Winding inner radius, mm & 322 & 180 \\
Magnetic yoke inner radius, $\mathrm{m}$ & 445 & 275 \\
Weight of yoke, t & 44 & 19 \\
Quadrupole outer dimensions: & & \\
width, mm & 1320 & 600 \\
height, $\mathrm{m}$ & 1800 & 1500 \\
\hline
\end{tabular}

The variant of quadrupole with the warm iron yoke and shell type of winding is visible but has a huge iron core weight and dimensions. Besides the cold mass block must be mounted with high precision relative to the iron yoke to eliminate magnetic decentering forces and magnetic field distortions. Nonlinear iron saturation effects have an influence on the field quality $(\sim 0.2 \%)$ and should be compensated by holes in the yoke or special correction system.

\section{B. Quadrupoles With Cold Iron Yoke}

The quadrupole with a cold iron yoke has a lower volume of superconductor because of low magnetic resistance for the return magnetic flux. But the limited width of cold yoke does not fully screen magnetic field. That is why small superconducting shielding coils should be mounted outside the yoke. Yoke saturation effects are also larger ( 0.3\%) than for a warm yoke design. The quadrupole cross-section and parameters are shown on Fig. 2 and Tab.III correspondingly.

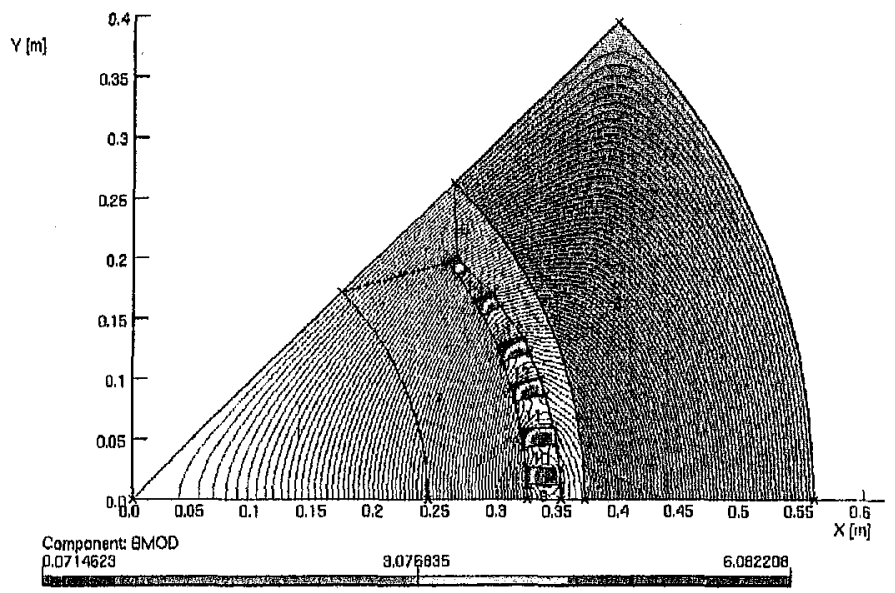

Fig.2 Flux lines distribution in the quadrupole with cold yoke 
TABLE III

QUADRUPOLES WITH COLD YOKE

\begin{tabular}{|c|c|c|}
\hline Parameter & Large Quadrupole & Small Quadrupole \\
\hline Total main current/pole, $\mathrm{kA}$ & 927 & 877 \\
\hline Total shielding current/pole, kA & -173 & -430 \\
\hline Magnetic flux density, $T$ & 3.185 & 2.74 \\
\hline at reference radius, $\mathrm{m}$ & 0.2413 & 0.1143 \\
\hline Maximum coil flux density, $\mathrm{T}$ & 6.08 & 7.8 \\
\hline Maximum iron flux density, $\mathrm{T}$ & 4.15 & 7.5 \\
\hline Energy of magnetic field, $\mathrm{MJ} / \mathrm{m}$ & 2.18 & 1.17 \\
\hline $\mathrm{Fx}, \mathrm{kN} / \mathrm{m}$ & 138 & -20 \\
\hline $\mathrm{Fy}, \mathrm{kN} / \mathrm{m}$ & -149 & -178 \\
\hline Winding inner radius, $\mathrm{m}$ & 322 & 180 \\
\hline Magnetic yoke inner radius, $\mathrm{mm}$ & 370 & 230 \\
\hline Weight of yoke $\mathrm{t} / \mathrm{m}$ & 18.6 & 1.1 \\
\hline Quadrupole outer radius, $\mathrm{mm}$ & 1280 & 640 \\
\hline
\end{tabular}

\section{Quadrupoles With Active Shield}

It is rather difficult to eliminate fringing magnetic fields when the magnet dimensions have strong limits. In this case the active shield winding looks to be a promising approach. Shielding windings are used in superconducting solenoids of MRI systems where needed to reduce magnet system weight. The shielding winding has an opposite direction of current relative to the main winding. It reduces the main field and, as a result, the larger quantity of supeconductor is needed. The quadrupole cross-section and parameters are shown on Fig.3 and Tab.IV correspondingly.

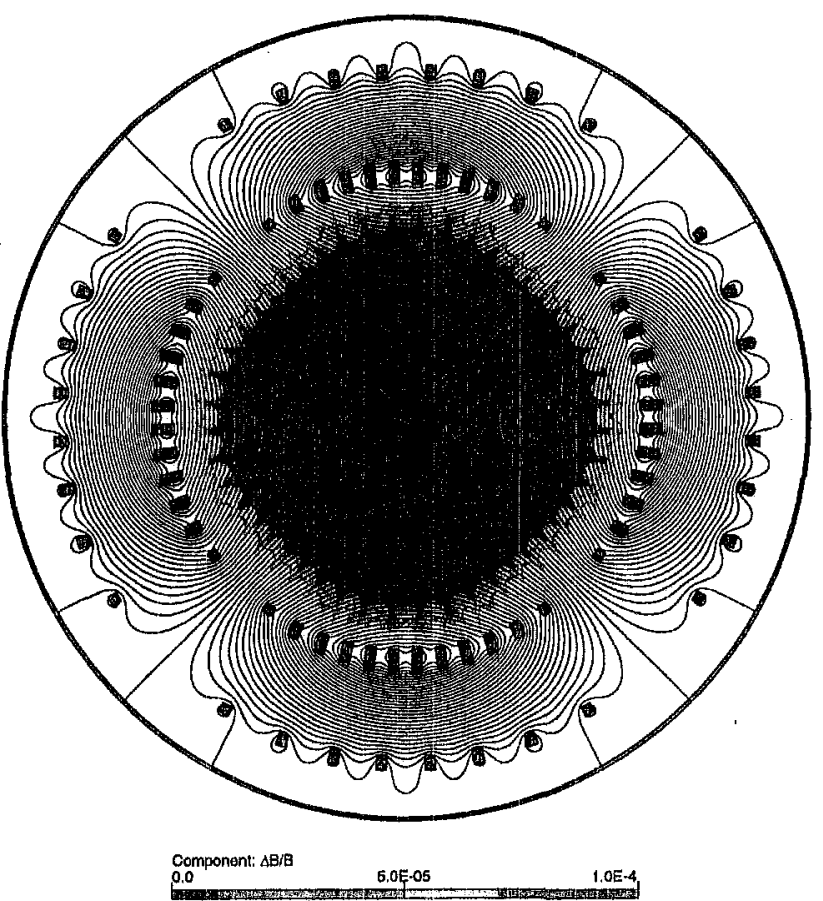

Fig.3 Flux lines and field quality distribution in the large bore quadrupole with active shield
Total ampere-turns in shielding winding can be calculated using a magnetic vector potential outside of the quadrupole shell type winding [6]:

$$
A_{z}(r, \theta)=\frac{2 \sqrt{3} \mu_{0} J_{0}\left(a_{2}^{4}-a_{1}^{4}\right)}{\pi r^{2}} \cos 2 \theta .
$$

where $J_{0}-$ current density, $a_{1}, a_{2}$ - coil inner and outer radiuses. The vector potential at full shielding effect should be equal to zero outside of the magnet system. Using (2), the dimensions of both windings and transforming the current density into the total current :

$$
I_{s h}=I_{w} \frac{a_{w 2}^{2}+a_{w 1}^{2}}{a_{s h 2}^{2}+a_{s h 1}^{2}}
$$

where $I_{s h}$ - the total current of shielding winding, $I_{w}-$ the total current of main winding, $a_{w 1}, a_{w 2^{-}}$inner and outer radiuses of main winding, ash - the same dimensions of shielding winding. The current in the shielding winding decreases as the square of average shielding winding radius. This means, that this radius should be as large as possible to increase the quadrupole efficiency.

TABLE IV

QUADRUPOLE WITH ACTIVE SCREEN

\begin{tabular}{lcc}
\hline Parameter & Large Quadrupole & Small \\
\hline Total main current/pole, kA & 1357 & 846 \\
Total shielding current/pole, kA & -532 & -382 \\
Magnetic flux density, T & 3.18 & 2.76 \\
$\quad$ at reference radius,m & 0.2413 & 0.1143 \\
Maximum main coil flux density, T & 7.24 & 7.22 \\
Maximum shield flux density, T & 2.5 & 3.5 \\
Energy of magnetic field, kJ/m & 3.12 & 1.13 \\
Magnetic forces/half-coil: & & \\
$\quad$ Fx, t/m & 28 & -1 \\
$\quad$ Fy, t/m & -264 & -176 \\
Main winding inner radius, mm & 322 & 180 \\
Shielding winding inner radius, mm & 560 & 290 \\
Quadrupole outer radius, mm & 1210 & 710 \\
& & \\
\hline
\end{tabular}

The quadrupole with an active shield has negligible fringing field. Very good field quality (Fig.3) can be obtained at all magnetic field levels. The total magnetic forces are selfcompensated and there are no coupling problems with adjacent magnets. There are also no iron saturation effects, hysteresis, or decentering effects. The main disadvantage of this magnet is It requires $\sim 70 \%$ more superconductor.

\section{RACETRACK TYPE COILS}

All previous variants of magnetic design are based on the shell type coils. Quadrupoles with racetrack type coils were also analyzed. These coils better accommodate magnetic forces and can be wound using $\mathrm{Nb}_{3} \mathrm{Sn}$ cable react-and-wind technology. The main problem is a larger cold mass width relatively to the shell type coil design. It is possible to significantly reduce the quadrupole width in the median plane 
by transforming the AHF optic system to work with skew quadrupoles $\left(45^{\circ}\right.$ rotated $)$. In this case, the racetrack design looks more reasonable (Fig. 4).

The racetrack main coils can be also combined with the shielding coils. Eight racetrack coils form both the main and shielding windings. Such configuration is more efficient for space saving and coil winding technology.

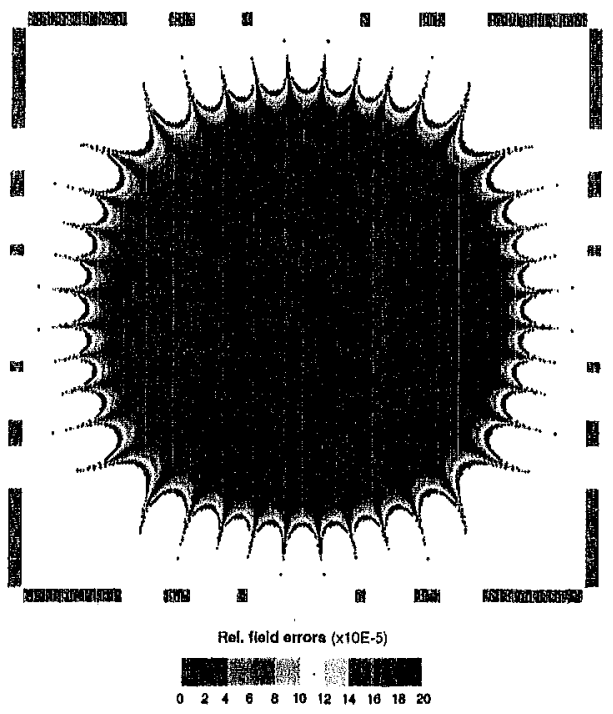

Fig. 4. Skew quadrupole with racetrack coils

\section{CHOICE OF SUPERCONDUCTOR}

The maximum field drives the choice of superconductor on the coils. As follows from the above tables, the flux density is in the range of $6 \mathrm{~T}-7.2 \mathrm{~T}$. At $6 \mathrm{~T}$ well-known $\mathrm{NbTi}$ cable can be used in both coils. $\mathrm{Nb}_{3} \mathrm{Sn}$ will be needed at higher fields. In any design the shielding coils will be wound from $\mathrm{NbTi}$ conductor. The field at the magnet ends is usually larger than in the central part and proper ends design is a goal for an optimization. $\mathrm{Nb}_{3} \mathrm{Sn}$ superconductor has larger temperature and magnetic field margin. The high intensity beam loss is unknown that will create additional heat load for the cryogenic system and reduce the maximum current density in the conductor. So, $\mathrm{Nb}_{3} \mathrm{Sn}$ conductor is the first candidate for the main winding manufacturing.

\section{Mechanical DESIGN}

The quadrupole mechanical design is based on previous experience of manufacturing an accelerator superconducting magnets at Fermilab. Existing equipment and tooling also direct the design to the more reasonable technology. The main difference of these quadrupoles is a large aperture and magnetic forces. Shell type coils wound into slots of stainless steel collar structure with wider cable side placed on the slot bottom. This provides stress management between coil blocks, more accurate cable position and equal prestress. Nevertheless, a sectional coil has on the surface higher flux density and proper optimization should be made between the stress management and the maximum coil field.

In the case of cold iron core the collar structure is thinner because the yoke with outer stainless steel skin can accommodate a significant fraction of the magnetic forces. The shielding coils should be manufactured from $\mathrm{NbTi}$ superconductor because of low magnetic field $\sim 3 \mathrm{~T}$ and forces on the outer radius of cold mass block.

The coil support should be precisely machined to determine the accurate coil position and magnetic field quality. $\mathrm{Nb}_{3} \mathrm{Sn}$ cable insulated with ceramic or fiberglass tape will be reacted inside this structure and vacuum impregnated with an epoxy. Some radial pre-stress should be applied to each block. The thick stainless steel teeth between the coil blocks will support the coil azimuthally. The support rigidity inside the welded skin of the helium vessel should be sufficient for the magnet operation.

Racetrack coils could be manufactured as a double pancake without an inner splice between layers. Reacted and impregnated $\mathrm{Nb}_{3} \mathrm{Sn}$ racetrack coils would be pre-loaded in the plane direction by side pushers during packaging of each pancake between the flat stainless steel sheets. The mechanical support structure around the racetrack coils will be designed to prevent coil motion under the Lorenz forces.

\section{CONCLUSION}

The conceptual analysis of large-bore quadrupoles for AHF confirmed the visibility of all the magnetic designs discussed above. The final choice of design for prototyping will be done later after beam losses analysis and cryostat design. The results, which follow from the magnetic analysis, are:

- outer dimensions of both types quadrupoles should be optimized simultaneously;

- warm iron core have larger: weight, dimensions, cold mass decentering forces and a complicated cold mass support system;

- both cold core or air core variants should have shield winding to eliminate fringing fields;

- $\quad \mathrm{Nb}_{3} \mathrm{Sn}$ superconductor is the preferable choice for the main winding and $\mathrm{NbTi}$ for the shield winding;

- racetrack type coils can simplify the winding;

- full scale racetrack coils can be used in the skew quadrupoles concept;

- some amount of a cold iron yoke is efficient but is limited by the necessity to correct iron saturation effects.

\section{REFERENCES}

[1] A.J.Jason, et al., "Beam-Distribution System for Multi-Axis Imaging at the Advanced Hydrotest Facility", Proceedings of the 2001 Particle Accelerator Conference, Chicago, 2001, pp. 3374-3378.

[2] G.E.Hogan, et al., "Proton Radiography", Proceedings of the 1999 Particle Accelerator Conference, 1999, pp. 579-583.

[3] C.T.Mottershead, J.D.Zumbro, "Magnetic Optics for Proton Radiography", Proceedings of the 1997 Particle Accelerator Conference, Vancouver, 1997, p.1397.

[4] S.Russenchuck, "A computer program for the design of superconducting accelerator magnets",Geneva, Switzerland, CERN AT/95-39, LHC Note 354, Sept.26, 1995.

[5] OPERA-2D User Guide: Vector Field Limited, VF-09-99-A3.

[6] H.Brechna, Superconducting Magnet Systems, Springer, Berlin, 1973. 